\title{
Comparison of Two Automated Methods for QT Interval Measurement
}

\author{
RE Gregg, S Babaeizadeh, DQ Feild, ED Helfenbein, JM Lindauer, SH Zhou \\ Advanced Algorithm Research Center, Philips Medical Systems, Andover, MA, USA
}

\begin{abstract}
In this paper we compared two methods of automated $Q T$ interval measurement on standard ECG databases: the Root-Mean-Square (RMS) lead combining method aimed at QT monitoring and the method of median of lead-by-lead QT interval measurements.

We used the PhysioNet PTB (N=548) and CSE measurement $(N=125)$ standard databases. Both have reference $Q T$ interval measurements from a group of annotators. The last 10 seconds of each PTB record was downsampled from 1000 sample per second (sps) and an amplitude resolution of $1 \mu \mathrm{V}$ to 500 sps and $5 \mu \mathrm{V}$ in order to match the CSE set. PTB records \#205 and \#557 were excluded due to ventricular paced rhythm and artifact, respectively. Twenty five cases were excluded from the CSE set to match the selection of cases for IEC algorithm testing (IEC 60601-2-51).

We processed all records using the Philips resting 12lead ECG algorithm to generate representative beats for $Q T$ interval measurement. The RMS method measures QRS onset and end of T on an RMS waveform constructed from 9 leads I, II, III and V1-V6. The lead-by-lead method takes the median QT interval across leads. The automated $Q T$ intervals by the RMS and lead-by-lead methods were compared to the reference manual QT measurements.

The mean difference between the lead-by-lead QT and the reference $Q T$ was $1.7 \pm 9.7 \mathrm{~ms}$ and $12.4 \pm 23.0 \mathrm{~ms}$ (mean sstandard deviation (SD)) for the CSE and PTB sets respectively. For the RMS method, the mean difference was $-2.8 \pm 11.1 \mathrm{~ms}$ and $10.3 \pm 20.9 \mathrm{~ms}$. F-tests indicate that the standard deviation between methods is not significantly different for the CSE set $(P=0.18)$ or the PTB set $(P=0.77)$.

The lead-by-lead and RMS methods perform similarly, leading to the conclusion that the choice between them should be based on considerations such as the number of leads available or computational efficiency.
\end{abstract}

\section{Introduction}

Global QT interval is one of the fundamental ECG measurements reported on virtually every 12 lead ECG. A longer than normal QT interval may indicate a congenital or acquired long QT condition [1-3]. The AHA/ACC practice guideline for ECG monitoring now includes a recommendation to monitor QT interval for the purpose of drug titration of drugs known to have a pro-arrhythmic effect [4]. If the QT interval lengthens by more than $60 \mathrm{~ms}$ after starting the drug or the QT interval extends beyond $500 \mathrm{~ms}$ the administration of the drug should be stopped or the dosage can be reduced.

We have previously presented Philips automated QT interval measurement algorithms for 12-lead ECG, Holter and ECG monitoring applications [5-10]. In the ambulatory Holter and patient monitoring ECG applications, the QT interval algorithm uses an RMS waveform from combined available high quality leads and measures QT interval on the RMS ECG. In the resting 12-lead ECG application, the global QT interval measurement is based on a lead-by-lead method. The open question is, of the two, which method is better ignoring the constraints of the application. In this paper, we compared the two automated methods and reported the results using the same ECG datasets.

\section{Study Population}

A comparison between automated methods aimed at either monitoring or ambulatory ECG versus 12-lead ECG is hampered by the gross difference in available ECG in each case. Ambulatory ECGs cannot be used to test the 12-lead QT algorithm because of low sample rate, narrow bandwidth and limited leads. For 12-lead ECG analysis, a sample rate of 500 sps and a bandwidth of 0.05 to $150 \mathrm{~Hz}$ are required. Ambulatory ECG recordings often have sample rates around 200 sps and a cut-off frequency of $40 \mathrm{~Hz}$. Only selected parts of the 12-lead analysis are possible with the small number of leads used in an ambulatory or monitoring recording. On the other hand, the sample rate, bandwidth and number of leads may be adequate for a 12-lead ECG to be used for an ambulatory analysis, but the 10 second recording is not long enough for even the learning period of the ambulatory and monitoring algorithms

The addition of the PTB set to the data publicly available at PhysioNet made this study possible because it has the features that allow direct comparison between ambulatory and 12 lead algorithms [11]. The PTB dataset consists of 549 records from 294 subjects with a sample 
rate of $1000 \mathrm{sps}$, an amplitude resolution of $1 \mathrm{uV}, 12$ scalar and 3 vector leads. In our analysis, the last 10 seconds of each record was down-sampled to a resolution of $500 \mathrm{sps}$ and $5 \mu \mathrm{V}$. Records \#205 and \#557 were excluded due to ventricular pacing and heavy artefact respectively. Reference manual QT intervals were collected from multiple annotators as part of the Computers in Cardiology 2006 challenge [12].

In general, automated ambulatory and 12 lead algorithms can be compared at the representative beat section of the algorithms. Most algorithms time-align and average many like-morphology beats together and extract features from that representative beat as discussed by Willems et al [13]. Through measurements made on the representative beat, results from longer ECG records and short 10 second ECG records can be compared. A second data set from the CSE project was used here [14]. The 100 ten-second records were selected from the total 125 according to the selection for computerized algorithm testing in the essential performance standard IEC 606012-51. Since the goal of the CSE and IEC effort was a minimum standard of measurement accuracy, problem cases such as atrial fibrillation were excluded because of undefined measurements. The resolution of the CSE 10 second records was 500 sps and $5 \mu \mathrm{V}$ per LSB.

\section{Methods}

The Philips resting 12-lead ECG algorithm was used to process all PTB and CSE records to produce representative beat signals. The dominant morphology beats were time-aligned and averaged to produce the representative beat. Custom MATLAB programs were used for the remaining processing. Statistical analysis was performed with the S-PLUS statistical software package.

In the lead-by-lead method of automated QT interval measurement, the QRS onset and end of $\mathrm{T}$ wave are determined separately. Although the earliest QRS onset and last $\mathrm{T}$ end are desired, the best accuracy and statistical stability is achieved using order statistics somewhere between the median and the maximum or minimum. Order statistics are a method of sorting a set of values and choosing one value from the set by order in the same way that the $50 \%$ value corresponds to the median. In our case, the QRS onset and $\mathrm{T}$ end times across leads I, II, III and V1-V6 are sorted. For global QRS onset, the 33\% value is chosen. The $50 \%$ value is chosen for the global $\mathrm{T}$ end. The Philips method was also applied for finding the end of $\mathrm{T}$ in each lead. Figure 1 shows an example of the QRS onset and $\mathrm{T}$ end measurements on each lead of a challenging case, s0330, from the PhysioNet PTB set. The third earliest value is chosen for the global QRS onset from all 9 individual values and the $5^{\text {th }} \mathrm{T}$ end value is chosen for the global end of $\mathrm{T}$.

In the RMS method, the global end of $\mathrm{T}$ is measured on an RMS ECG based on combination of leads I, II, III and V1-V6. The same Philips method is applied for identification of the $\mathrm{T}$ end.

Without any correction, severe ST deviation causes errors with the RMS method. Large ST deviation may cause the RMS waveform to exhibit an apparent $T$ wave peak due to ST deviation that is taller than the true T wave peak. The same case is shown in Figures 1 and 2 . The chest leads have large ST depression with a small opposite polarity $\mathrm{T}$ wave. Each chest lead therefore has a zero crossing between the ST depression and the peak of the $\mathrm{T}$ wave. The dash-dot line of Figure 2 represents the RMS curve with no correction for the ST deviation. The zero crossings result in a null in the RMS curve. The end of $\mathrm{T}$ search would identify this null as the end of $\mathrm{T}$ as if the next peak is due to a $U$ wave. The solid line of Fig. 2 shows the RMS curve after correction. The large hump in the dotted line is the false $\mathrm{T}$ wave peak in the uncorrected RMS signal. The correction to the RMS signal involves adding an offset to each lead before calculating the RMS curve to prevent the ST-T zero crossing. The correction for all leads is then subtracted out of the RMS curve to bring the baseline back to zero.

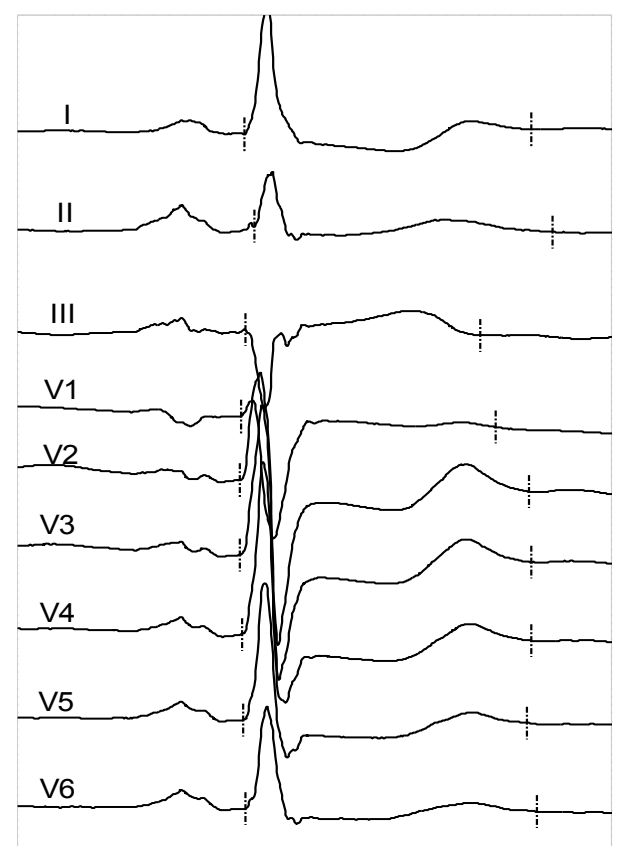

Figure 1. Representative beat, PhysioNet PTB case s0330, displayed in a high gain cascade format showing the automated QRS onset and $\mathrm{T}$ end locations used to calculate the lead-by-lead global QT. 


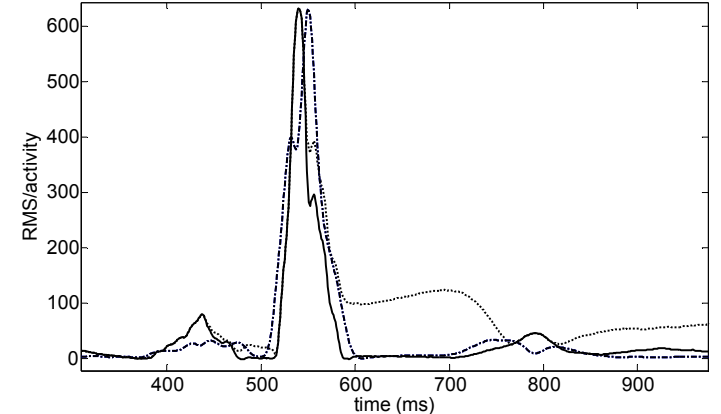

Figure 2. RMS waveform (solid line) of the same ECG signal as shown in Fig. 1. The uncorrected RMS waveform in dotted-line is calculated by combining leads I, II, III, and V1-V6. The dash-dotted line illustrates the corresponding activity.

In the RMS method, QRS onset is determined from an activity signal rather than the RMS signal. The activity function is defined below by equation 1. It is the absolute value first difference signal summed across leads. Since the high frequency content of the QRS part of the complex far exceeds the high frequency content of $\mathrm{P}$ and especially $\mathrm{T}$ waves, the peaks of the QRS part of the activity function are large compared to the rest of the complex. Although the activity function is susceptible to high frequency noise because of the use of first differences, beat-averaging before the activity function calculation and lowpass filtering of the activity function reduce the noise susceptibility.

$$
\text { (1) } y i=\sum_{j=1}^{L}|x j i-x j(i-1)|
$$

\section{Results}

The differences between automated measurement of QT interval and the reference manual QT interval are summarized below in Table 1. The differences between automated and manual QT measurements are characterized by mean difference (automated minus manual), and the standard deviation.

Table 1. QT differences, automated minus manual, for the two automated methods on both the CSE and PTB sets. $\mathrm{SD}=$ standard deviation.

\begin{tabular}{lcccc}
\hline $\begin{array}{l}\text { Automated } \\
\text { algorithm }\end{array}$ & \multicolumn{2}{c}{$\begin{array}{c}\text { CSE } \\
(\mathrm{N}=100)\end{array}$} & \multicolumn{2}{c}{$\begin{array}{c}\text { PTB } \\
(\mathrm{N}=546)\end{array}$} \\
\hline & $\begin{array}{c}\text { Mean } \\
(\mathrm{ms})\end{array}$ & $\begin{array}{c}\text { SD } \\
(\mathrm{ms})\end{array}$ & $\begin{array}{c}\text { Mean } \\
(\mathrm{ms})\end{array}$ & $\begin{array}{c}\text { SD } \\
(\mathrm{ms})\end{array}$ \\
\hline Lead-by-lead & 1.7 & 9.7 & 12.4 & 23.0 \\
RMS & -2.8 & 11.1 & 10.3 & 20.9 \\
\hline
\end{tabular}

The striking feature of the test results is the similarity of standard deviation of QT measurement differences and the approximate $3 \mathrm{~ms}$ mean difference between the methods on the two data sets. The F-test indicate that the difference in standard deviation between the methods is not significant for the CSE set $(\mathrm{p}=0.18)$ or the PTB set $(p=0.77)$. For the CSE set, the paired T-test results in a difference of $4.5 \mathrm{~ms}$ between methods with a 95\% confidence interval of 1.7 to $7.3 \mathrm{~ms}$. For PTB, the difference between automated methods is $2.0 \mathrm{~ms}$ with a confidence interval of 0.2 to $3.9 \mathrm{~ms}$.

The median and interquartile range (IQR) are used to measure the central tendency and variation in QT interval differences without undue effect of outliers. The median and IQR values in Table 2 show a similar result as mean and standard deviation. The lead-by-lead method has slightly less variation for the CSE set and the RMS method has slightly less variation for the PTB set. The difference between median values is close to the difference in means.

Table 2. Median and interquartile range (IQR) of QT differences for CSE and PTB sets.

\begin{tabular}{lcccc}
\hline $\begin{array}{l}\text { Automated } \\
\text { algorithm }\end{array}$ & \multicolumn{2}{c}{$\begin{array}{c}\text { CSE } \\
(\mathrm{N}=100)\end{array}$} & \multicolumn{2}{c}{$\begin{array}{c}\text { PTB } \\
(\mathrm{N}=546)\end{array}$} \\
\hline & $\begin{array}{c}\text { Median } \\
(\mathrm{ms})\end{array}$ & $\begin{array}{c}\text { IQR } \\
(\mathrm{ms})\end{array}$ & $\begin{array}{c}\text { Median } \\
(\mathrm{ms})\end{array}$ & $\begin{array}{c}\text { IQR } \\
(\mathrm{ms})\end{array}$ \\
\hline Lead-by-lead & 1.0 & 9.5 & 11.0 & 19.5 \\
RMS & -3.0 & 12.3 & 9.0 & 19.0 \\
\hline
\end{tabular}

\section{Discussion}

The mean difference between methods has a somewhat consistent small bias of approximately $3 \mathrm{~ms}$ for both data sets according to the paired T-tests. Both methods are close to zero mean performance for the CSE set while both methods measure QT long by approximately $10 \mathrm{~ms}$ compared to the manual annotation for the PTB set. This suggests a potential bias in the reference QT measurements between the CSE and PTB sets. Bortolan trained on the CSE and tested on the PTB set and also reported a bias in reference QT measurements with a much larger value of $25 \mathrm{~ms}$ [13]. This bias could easily be explained by the fact that the PTB annotators concentrated on lead II while the CSE annotators used 8 available leads I, II and V1 - V6 $[14,15]$.

From the results in Table 1 and Table 2, it is difficult to choose one automated method over the other since each method is slightly better for one of the two sets but not both. The lead-by-lead method results in better ontarget performance and low variation for the CSE set while the RMS method has a smaller mean difference and measurement variation on the PTB set. Each data set has 
advantages and disadvantages. The CSE group collected ECGs to represent a general hospital population. The PTB patient selection is composed of a high proportion of myocardial infarction (MI) cases and a smaller group of normal subjects. The CSE set was annotated on multiple leads by multiple annotators very carefully and the method was well documented [16]. All primary leads were used and the annotation was performed on high resolution ECG which has been shown to affect the QT measurement [17]. The annotators of the PTB database were instructed to concentrate on only lead II. On the other hand, the PTB set has 550 cases compared to the CSE set of 125 cases. The effect of larger population can be seen in the tight confidence interval for the mean QT difference for the PTB set. Since the CSE set was annotated across all leads at a high gain, we weigh the CSE results more heavily and therefore believe our automated methods to be on-target even though they exhibit a $10 \mathrm{~ms}$ bias according to the PTB set.

\section{Conclusion}

In conclusion, the two automated methods presented produce comparable results so that either method could be chosen. Both methods perform well. The lead-by-lead method requires more leads for the application of order statistics to make sense. The RMS method can use just a single lead. For this reason, both the lead-by-lead and RMS methods can be used for 12 lead resting ECG analyses. However, the lead-by-lead method does require more computational power. In real-time monitoring and ambulatory ECG applications with a limited number of leads available and restrained computational power, the RMS method works well and should be used.

\section{References}

[1] Moss A. QTc prolongation and sudden cardiac death. JACC 2006;47:2:368-369.

[2] Towbin J, Friedman R. Prolongation of the QT interval and the sudden infant death syndrome. $N$ Engl $J$ Med 1998;338:1760-1761.

[3] Priori SG, Schwartz PJ, Napolitano C, et al. Risk stratification in the long-QT syndrome. N Engl J Med 2003;348:1866-1874.

[4] Drew BJ, Califf RM, Funk M, et. al. Practice standard for Electrocardiographic monitoring in hospital settings. An American Heart Association Scientific Statement from the councils on cardiovascular nursing, clinical cardiology and cardiovascular disease in the young. Circulation 2004,110:2721-2746.

[5] Lindauer JM, Gregg RE, Helfenbein ED, Shao M, Zhou SH. Global QT measurements in the Philips 12-lead algorithm. J Electrocardiol, 2005, Vol. 38: p S90.
[6] Feild DQ, QT measurement performance in a Holter application. J Electrocardiol 2005, 38:S34.

[7] Helfenbein ED, Zhou SH, Lindauer JM, Feild DQ, Gregg RE, Wang JY, Kresge SS, Michaud FP. An algorithm for continuous real-time QT interval monitoring. J Electrocardio 2006;39:S123-S127.

[8] Helfenbein ED, Zhou SH, Feild DQ, Lindauer JM, Gregg RE, Wang JY, Kresge SS, Michaud FP. Performance of a continuous real-time QT interval monitoring algorithm for the critical-care setting. Computers in Cardiology 2006 , 33:697-700.

[9] Helfenbein ED, Ackerman MJ, Rautaharju PM, Zhou SH, Gregg RE et al.: An algorithm for QT interval monitoring in neonatal intensive-care units. J Electrocardiol 2007 (supp) (in press)

[10] Zhou SH, Helfenbein ED, Lindauer JM, Gregg RE, Feild DQ: Philips QT interval measurement algorithms for diagnostic, ambulatory and patient monitoring ECG applications. ANE, 2007 suppl. (in press)

[11] Goldberger AL, Amaral LAN, Glass L, Hausdorff JM, Ivanov PCh, Mark RG, Mietus JE, Moody GB, Peng CK, Stanley HE. PhysioBank, PhysioToolkit, and PhysioNet: Components of a New Research Resource for Complex Physiologic Signals. Circulation 2000, 23:e215-e220.

[12] Moody GB, Koch H, Steinhoff U. The PhysioNet/Computers in Cardiology Challenge 2006: QT interval measurement. Computers in Cardiology (33) 2006.

[13] Bortolan G. Algorithmic testing for QT interval measurement. Computers in Cardiology 2006, 33:365-368.

[14] Willems JL, Arnaud P, van Bemmel JH, Bourdillon PJ, Degaini R, et al. Establishment of a reference library for evaluating computer ECG measurement programs. Comput. Biomed Research 1985, 18:439-457.

[15] Willems JL, Arnaud P, van Bemmel JH, Degani R, Macfarlance PW, Zywietz C. Common standards for quantitative electrrcardiography: goals and main results. Methods of Information in Medicine 1990, 29:263-271.

[16] Commission of the European Communities, Medical and Public Health Research (Willems JL CSE Project Leader): Common Standards for Quantitative Electrocardiography, CSE Multilead Atlas. CSE Ref. 88-04.15 Acco Publ, Leuven Belgia, 1988.

[17] Murray A, McLaughlin NB, Bourke JP, Doig JC, Furniss SS, Campbell RWF. Errors in manual measurement of QT intervals, Br Heart J 1994, 71:386-390.

Address for correspondence.

Richard Gregg

Advanced Algorithm Research Center (AARC)

Philips Medical Systems

3000 Minuteman Drive, Mail stop 0220

Andover, MA 01810

USA

rich.gregg@philips.com 